\title{
Inferior Parietal Perfusion, Lateralization, and Neuropsychological Dysfunction in Alzheimer's Disease
}

\author{
John G. Keilp, Gene E. Alexander, Yaakov Stern, \\ AND ISAK PROHOVNIK
}

Department of Brain Imaging, New York State Psychiatric Institute, and the Departments of Neurology, Radiology, and Psychiatry, College of Physicians and Surgeons,

Columbia University

The severity of inferior parietal perfusion deficits in Alzheimer's disease (AD) is strongly associated with global intellectual decline. The relationship to specific losses of neuropsychological functioning, however, is less clear, as is the relative importance of the side (left vs. right) of hemispheric deficit. In this study, 53 patients with probable $\mathrm{AD}$ and 35 elderly controls received both a resting ${ }^{133} \mathrm{Xe} \mathrm{rCBF}$ measurement and neuropsychological examination. AD patients demonstrated the expected bilateral deficits in inferior parietal perfusion, as well as impairment on measures of mental status, intelligence, verbal and visual memory, attention, language, and construction abilities. The severity of this bilateral parietal deficit, in turn, was associated with virtually all of these AD-related neuropsychological impairments, most strongly with declining Performance IQ. Left-sided deficits correlated better with overall declines in IQ, as well as with declining attention and language fluency. Right-sided deficits, on the other hand, correlated best with declines in mental status and-paradoxically-verbal memory and contributed independently to declines in Full Scale and Performance IQ. In terms of the number and strength of their association to neuropsychological measures, left-sided deficits appear much more predictive of cognitive decline in AD. Right-sided deficits, however, may be most important for predicting aspects of performance skill that are only indirectly assessed in standard paper-and-pencil format. Overall, it appears that both sides make significant, but independent contributions to general functional decline in $\mathrm{AD}$, but that left-sided deficits are more closely associated with cognitive decline as measured by most standard neuropsychological measures. () 1996 Academic Press, Inc.

This work was supported by NIH Grants AG05433 and AG10638. We thank Amy Wu, M.D., and Edward Huey, B.A., for their assistance in the completion of this project. We also thank Drs. Richard Mayeux and Davangere Devanand for permission to study their patients. Address reprint requests to Dr. Keilp, at Unit 64, New York State Psychiatric Institute, 722 West 168th Street, New York, NY 10032. 


\section{INTRODUCTION}

Deficits in inferior parietal perfusion are now well established as an indicator of Alzheimer's disease (AD; Friedland, Budinger, \& Ganz, 1983; Jagust, Budinger, \& Reed, 1987; Prohovnik, Mayeux, Sackheim, Smith, Stern, \& Alderson, 1988; Holman, Johnson, \& Gerada, 1992). The severity of these deficits, in turn, correlates with measures of mental status, as demonstrated in studies using planar ${ }^{133} \mathrm{Xe}$ techniques (Prohovnik et al., 1988), PET (DeLeon, Ferris, \& George, 1983; Frackowiack, Pozzilli, \& Legg, 1981), and SPECT (DeKosky, Shih, Schmitt, Coupal, \& Kirkpatrick, 1990; Jagust et al., 1987; Johnson, Holman, Mueller, Rosen, English, Nagel, \& Growdon, 1988; Montaldi, Brooks, McColl, Wyper, Patterson, Barron, \& McCulloch, 1990; Robert, Migneco, Darcourt, Ricq, Aubin, Bonhomme, Pringuey, Lapalus, \& Darcourt, 1992). More recently, we have found that inferior parietal deficits strongly predict the estimated decline in IQ that occurs during the disease (Keilp \& Prohovnik, 1995), suggesting that, properly quantified, these deficits may be used as an index of disease severity in individual cases. Questions remain, though, about the exact neuropsychological correlates of these deficits and the significance of their laterality. Karbe, Kertesz, Davis, Kemp, Prato, and Nicholson (1994), for example, have shown that left-sided deficits are most important for predicting cognitive loss, when this cognitive loss is measured by the Mattis Dementia Rating Scale. Robert et al. (1992), on the other hand, found that right-sided deficits best predict mental status decline when it is measured by the Mini-Mental State Exam. The purpose of this study was to examine the association of inferior parietal deficits measured using ${ }^{133} \mathrm{Xe}$ methods to a broad array of neuropsychological functions that are disturbed in $\mathrm{AD}$, to determine if these deficits are associated with general decrements in intellectual functioning or some more specific, core neuropsychological impairment. In addition, the relative contribution of right- and left-sided deficits to these associations was also examined, in order to characterize the contribution of each to general cognitive decline.

\section{METHODS}

Subjects. Fifty-three elderly men and women with probable Alzheimer's disease were diagnosed by NINCDS-ADRDA criteria (McKhann, Drachman, Folstein, Katzman, Price, \& Stadlan, 1984) according to procedures previously described (Alexander, Prohovnik, Stern, \& Mayeux, 1994a). A control sample of 35 healthy elderly men and women were also recruited and administered the same measures as patients, including the modified Mini-Mental State Examination (mMMS; Mayeux, Stern, Rosen, \& Leventhal, 1981), Blessed Dementia Rating Scale (BDRS; Blessed, Tomlinson, \& Roth, 1968), a neuropsychological battery (described below), and rCBF measurement (also described below). Demographic and clinical severity characteristics of these samples are provided in Table 1. With regard to the specific measures included in this table, the Occupational Status rating is the 6-point scale used by Wechsler in the standardization of the WAIS-R ( 1 = lowest, $6=$ highest: Wechsler, 1981). The estimate of Premorbid IQ is based on a demographic formula developed by Barona et al. (1984) which uses the variables of age, sex, race, education, occupational status, and region of residence 
TABLE 1

Sample Characteristics

\begin{tabular}{lcc}
\hline & AD patients & Controls \\
\hline$N$ & 53 & 35 \\
Age & $68.6 \pm 10.0$ & $64.6 \pm 7.8^{*}$ \\
Sex ratio & $60.4 \%$ Female & $62.9 \%$ Female \\
& $39.6 \%$ Male & $37.1 \%$ Male \\
Racial composition & $88.7 \%$ White & $91.4 \%$ White \\
& $9.4 \%$ Black & $5.7 \%$ Black \\
Native language & $1.9 \%$ Hispanic & $2.9 \%$ Asian \\
Education & $76.9 \%$ English & $85.7 \%$ English \\
Occupation rating & $12.9 \pm 4.7$ & $15.2 \pm 2.6^{* *}$ \\
Estimated premorbid IQ & $4.6 \pm 1.4$ & $5.4 \pm 1.2 * * *$ \\
Age of onset & $105.8 \pm 10.7$ & $113.4 \pm 6.6 \dagger$ \\
Duration of illness & $64.1 \pm 10.2$ & - \\
Modified Mini-Mental State Exam & $4.2 \pm 2.1$ & - \\
Estimated Folstein Mini-Mental Score & $31.6 \pm 8.7$ & $54.4 \pm 1.6 \ddagger$ \\
Blessed Dementia Rating Scale & $15.9 \pm 4.8$ & $28.6 \pm 0.9 \ddagger$ \\
Systolic blood pressure & $9.7 \pm 3.7$ & $.34 \pm .53 \S$ \\
Diastolic blood pressure & $134.0 \pm 17.4$ & $128.6 \pm 18.1$ \\
End-tidal $p \mathrm{CO}_{2}$ & $75.2 \pm 13.3$ & $76.3 \pm 8.5$ \\
\hline
\end{tabular}

$$
\begin{aligned}
* t[86] & =1.74, p=.085 . \\
* * t[85] & =2.64, p=.010 . \\
* * * t[85] & =2.86, p=.005 . \\
\dagger t[85] & =3.75, p<.001 . \\
\ddagger t[86] & =15.28, p<.001 . \\
\S t[83] & =14.11, p<.001 .
\end{aligned}
$$

to estimate WAIS-R IQ. Its accuracy is reflected in the degree to which it is able to predict the actual, current WAIS-R IQ scores of the controls (actual mean WAIS-R IQ in controls $=114.4 \pm 15.3$, paired $-t[20]=.77, p=.449)$. An estimated score for the Folstein MiniMental State Exam (Folstein et al., 1981) is also provided in the table. This estimated score is based on a linear conversion of the mMMS developed in our laboratory (MMS $=[\mathrm{mMMS}$ $-3] / 1.8 ; r=.98$ for 124 joint administrations; Prohovnik, Keilp, \& Huey, unpublished data) and is computed in order to compare measures of mental status to recently promulgated ageand education-stratified norms (Crum et al., 1993), which are unavailable for the mMMS.

The AD patients in this sample have a mean age of onset in their mid-sixties, a wide range of duration of illness, and mMMS and BDRS scores that suggest dementia of moderate severity. Demographically, however, they differ in some respects from controls. Controls are slightly younger and have significantly more education, higher lifetime occupational status (managerial/clerical vs. skilled workers), and higher estimated "premorbid"' IQ.

Neuropsychological assessment. Subjects were administered either a long or abbreviated battery of neuropsychological tests. The long battery incorporated tests of mental status (mMMS) and general intelligence (WAIS-R), as well as more detailed assessments of memory (Buschke Selective Reminding Test), attention (Cancellation Tasks using the number 6, a diamond shape, and the consonant trigram TMX as targets: Sano et al., 1984), language (12 subtests from the Boston Diagnostic Aphasia Exam, including Body Part Identification, Commands, Complex Ideational Material, Repeating Phrases: High Probability, Repeating Phrases: 
Low Probability, Word Repetition, Responsive Naming, Oral Reading, Word-Picture Matching, Reading Sentences and Paragraphs, Primer Level Dictation, and Confrontation Naming; as well as tests of Letter Fluency [CFL], Category Fluency [Animal Naming]), visual memory (Benton Visual Retention Test, Administration M [matching]), and construction abilities (Rosen Drawing Test: Rosen, 1981). This full battery was administered to $54.7 \%$ of AD patients and $60.0 \%$ of controls. The abbreviated battery, including only the tests of mental status, attention, and memory, was administered to the remaining subjects. In a number of cases, subject's refusal to perform a test, confusion, or agitation resulted in its being dropped from a testing session. In addition, the attention tests (Cancellation) were not initially included in the battery for 12 subjects who were administered the long battery.

All neuropsychological test scores were adjusted for either age- and/or education-stratified normative expectations and expressed as either "decline", scores (obtained score minus norm; used for both the estimated MMS and WAIS-R IQ, where the test metric is widely recognized) or $Z$ scores (obtained score minus normative mean divided by standard deviation of norm group). IQ subtest decline scores are based on the difference between obtained subtest scores and an expected score based on a translation of the estimated IQ into an average subtest score. The purpose of these adjustments was to correct for demographic confounds on test score data (Berkman, 1986; Kittner, White, Farmer, Wolz, Kaplan, Moes, Brody, \& Feinleib, 1986) which we had previously shown can exert a powerful influence on associations with imaging findings (Stern, Alexander, Prohovnik, \& Mayeux, 1992). Among 60 correlations computed between test score data and perfusion indices here, 44 increased in magnitude after normative adjustment, and 13 additional significant associations that were not observed with raw test scores were found.

Published norms were available for the Folstein MMS, WAIS-R, Buschke SRT, Boston Diagnostic Aphasia Exam (BDAE), Semantic Fluency, and Category Fluency tests. For the Cancellation Tasks, Benton VRT (administration M), and Rosen Drawing Task, normative means and standard deviations were obtained from age-stratified $(\leq 59,60-69, \geq 70)$ subgroups of the control sample. For conciseness, $Z$ scores from BDAE subtests were summed and averaged in order to provide a single index of language disturbance for analyses here.

Because normative corrections for the BDRS were not available, it is not included in the analyses below. Like most uncorrected scores, the BDRS score was uncorrelated with perfusion measures in nearly all analyses, except among short duration of illness patients ( $\leq 3$ years), where it was strongly associated with the index of parietal perfusion deficit used here $(r=$ $-.60, p=.008, n=18)$.

Regional cerebral blood flow assessment. Subjects' rCBF was assessed using the ${ }^{133} \mathrm{Xe}$ inhalation technique. Data reported here are from the first in a series of scans, conducted under resting conditions (subjects lying still, with eyes closed in a darkened, quiet room). Scanning procedures used in our laboratory have been described previously (Prohovnik, 1988). Planar counts in 32 brain regions were obtained with $\mathrm{NaI}$ (Tl) scintillation detectors sampling 16 cortical regions in each hemisphere, using a commercial device (NOVO Cerebrograph 32c). The principal measure of perfusion was the Initial Slope Index (ISI), calculated using the M2 four-compartment model (Prohovnik, Knudson, \& Risberg, 1983, 1985). M2 ISI has previously been shown to be highly reliable in low flow conditions, as expected here, and relatively immune to atrophy.

End-tidal $p \mathrm{CO}_{2}$, blood pressure and respiratory rate were recorded using a fully computerized system. Blood pressure and $p_{\mathrm{e}} \mathrm{CO}_{2}$ values were all within normal range for this age group and did not differ between patients and controls (see Table 1). To minimize the influence of respiration artifacts, absolute perfusion estimates were conservatively corrected to a $p_{\mathrm{e}} \mathrm{CO}_{2}$ value of $37 \mathrm{~mm} \mathrm{Hg}$ by $2 \% / \mathrm{mm} \mathrm{Hg}$ (Maximillian, Prohovnik, \& Risberg, 1980). Whole-cortex mean flow was computed as the average of the 32 adjusted regional cortical values. Inferior parietal perfusion was characterized in terms of the Parietal Index (PI), computed by expressing flow to inferior parietal detectors (P1 and P3 bilaterally), which are strongly affected by the disease, as a percentage of flow to perirolandic (C1) and occipital (O2) detectors, which are relatively preserved. Mean flow is included here because it is reduced in AD, but not specifi- 
cally an indicator of the disease. The PI, in contrast, is linked more directly with AD, in that it can be used to reliably discriminate probable cases from a variety of other groups, including normal controls (Alexander, Prohovnik, Stern, \& Mayeux, 1994a), elderly major depressives (Sackeim, Prohovnik, Moeller, Mayeux, Stern, \& Devanand, 1993), and cases of vascular dementia (Prohovnik, Alexander, Tatemichi, 1991).

Statistical analyses. Normalized data from neuropsychological assessments, as well as rCBF data, were first compared between AD patients and controls, to establish the characteristics of the patient sample. Neuropsychological data were compared using two-way analysis of covariance techniques, with diagnostic status and sex as factors and age, education, and occupational status as covariates. Though these variables are partially controlled for in the process of normatively adjusting neuropsychological scores initially, this covariance method allowed for extracting residual effects not controlled for in normative corrections, as well as a means of examining the effectiveness of the normative corrections. Data from rCBF measurement was compared between groups in a similar fashion, in order to adjust for group differences in demographics.

Following group comparisons, correlations between normatively adjusted neuropsychological data and bilateral $\mathrm{rCBF}$ measures were computed in the patient group only to determine the association between them.

Stepwise regression was then used in an exploratory manner to generate a parsimonious set of neuropsychological predictors of $\mathrm{rCBF}$ deficits within the AD patient subject group. The purpose of this step was to find that subset of tests that best characterized the variance in $\mathrm{rCBF}$ measures related to declining cognitive functioning. Two stepwise procedures were run: one using all test scores, but only those subjects who were administered the long battery, and another using all subjects and those test scores from the short battery. Mean substitution was used in this analysis (and in this analysis alone) to compensate for missing data from each battery, so that it would be possible to compare among the tests and find those that best predicted inferior parietal $\mathrm{rCBF}$.

Finally, lateralized perfusion to inferior parietal regions was examined in relation to neuropsychological scores, in order to determine if the extent of deficit on left vs. right sides was differentially related to cognitive performance. These associations were expressed as partial correlations, controlling for the effects of both the opposite-sided index and the overall mean flow.

\section{RESULTS}

Neuropsychological performance in AD patients and controls. Normalized neuropsychological performance data for $\mathrm{AD}$ patients and controls are presented in Table 2. Even after accounting for sex effects and statistically adjusting for group differences in age, education, and occupational status, AD patients and controls differ significantly on all measures administered. No main effects for sex are observed, and only a single sex by diagnosis interaction is found for the estimated decline in MMS score $(F[1,80]=$ $5.41, p=.023)$. This effect, however, is related to slightly lower than expected scores among male controls, rather than any significant differences between male and female patients $(t[50]=1.61, p=.114)$.

Despite the use of normatively adjusted test scores, covariates were, in some cases significant. Residual age effects are found for Buschke LTR-B score $(F[1,77]=4.56, p=.038)$ and Recognition score $(F[1,75]=4.04$, $p=.048)$ and for the Cancellation task using the number Six $(F[1,65]=$ $12.09, p=.001)$ and Diamond shape $(F[1,64]=6.09, p=.016)$ as targets. Education effects were found for Letter Fluency $(F[1,39]=9.63, p=.004)$ 
TABLE 2

Patient/Control Comparisons on Neuropsychological Measures

\begin{tabular}{|c|c|c|c|c|}
\hline & AD patients & Controls & $N^{\prime} \mathrm{s}$ & $\mathrm{F}(p$ value $)$ \\
\hline Mini-Mental State Decline ${ }^{a}$ & $-11.4 \pm 4.9$ & $0.1 \pm .94$ & $52 / 35$ & $197.16(<.001)$ \\
\hline \multicolumn{5}{|c|}{ Buschke Selective Reminding Test (SRT) } \\
\hline Total & $-5.6 \pm 2.6$ & $0.2 \pm .78$ & $50 / 34$ & $162.40(<.001)$ \\
\hline LTR & $-3.9 \pm 1.6$ & $0.3 \pm .65$ & $50 / 34$ & $227.20(<.001)$ \\
\hline LTR-B & $-2.6 \pm 1.1$ & $0.1 \pm .65$ & $50 / 34$ & $208.85(<.001)$ \\
\hline Intrusions & $0.8 \pm 2.6$ & $-0.3 \pm .58$ & $50 / 34$ & $8.03(.006)$ \\
\hline Delay & $-4.3 \pm 2.0$ & $0.2 \pm .85$ & $48 / 34$ & $154.29(<.001)$ \\
\hline Recognition & $-24.4 \pm 16.6$ & $0.0 \pm .89$ & $48 / 34$ & $55.65(<.001)$ \\
\hline \multicolumn{5}{|l|}{ Cancellation Tasks } \\
\hline Six & $-3.0 \pm 3.5$ & $0.0 \pm .97$ & $39 / 33$ & $26.36(<.001)$ \\
\hline Diamond & $-4.7 \pm 6.4$ & $0.0 \pm .97$ & $38 / 33$ & $24.22(<.001)$ \\
\hline TMX & $-5.8 \pm 4.8$ & $0.0 \pm 1.3$ & $38 / 33$ & $46.38(<.001)$ \\
\hline IQ decline, full scale ${ }^{a}$ & $-28.0 \pm 15.5$ & $1.9 \pm 11.8$ & $27 / 21$ & $59.81(<.001)$ \\
\hline IQ decline, verbal scale & $-22.4 \pm 14.3$ & $4.0 \pm 9.2$ & $27 / 21$ & $58.24(<.001)$ \\
\hline IQ decline, performance scale & $-32.3 \pm 15.2$ & $-2.3 \pm 14.1$ & $27 / 21$ & $59.78(<.001)$ \\
\hline \multicolumn{5}{|l|}{ IQ Subtests (Decline) $)^{a}$} \\
\hline Information & $-4.2 \pm 3.3$ & $0.5 \pm 1.6$ & $27 / 21$ & $36.66(<.001)$ \\
\hline Digit Span & $-3.7 \pm 3.7$ & $-1.0 \pm 2.6$ & $27 / 21$ & $15.76(<.001)$ \\
\hline Vocabulary & $-2.7 \pm 2.7$ & $1.1 \pm 1.9$ & $27 / 21$ & $31.77(<.001)$ \\
\hline Arithmetic & $-5.3 \pm 3.4$ & $1.1 \pm 2.5$ & $28 / 21$ & $42.51(<.001)$ \\
\hline Comprehension & $-4.4 \pm 4.1$ & $1.1 \pm 2.5$ & $27 / 21$ & $38.66(<.001)$ \\
\hline Similarities & $-4.7 \pm 2.8$ & $-0.7 \pm 2.5$ & $27 / 21$ & $33.24(<.001)$ \\
\hline Picture Completion & $-5.9 \pm 3.5$ & $-0.7 \pm 2.8$ & $27 / 21$ & $49.20(<.001)$ \\
\hline Picture Arrangement & $-5.6 \pm 3.5$ & $-0.3 \pm 2.6$ & $27 / 21$ & $44.60(<.001)$ \\
\hline Block Design & $-6.0 \pm 3.6$ & $-0.5 \pm 3.5$ & $28 / 21$ & $42.35(<.001)$ \\
\hline Object Assembly & $-5.1 \pm 3.6$ & $-2.0 \pm 2.9$ & $28 / 21$ & $17.73(<.001)$ \\
\hline Digit Symbol & $-6.7 \pm 3.8$ & $-0.8 \pm 2.2$ & $28 / 21$ & $53.11(<.001)$ \\
\hline $\begin{array}{l}\text { Boston Diagnostic Aphasia Exam } \\
\text { (average } Z \text { score/subtest) }\end{array}$ & $-5.0 \pm 4.6$ & $-0.1 \pm 0.4$ & $23 / 20$ & $30.56(<.001)$ \\
\hline Letter fluency (CFL) & $-1.2 \pm .98$ & $0.8 \pm 1.13$ & $25 / 21$ & $18.12(<.001)$ \\
\hline Category fluency (animals) & $-2.2 \pm 1.0$ & $0.6 \pm 1.2$ & $25 / 21$ & $47.18(<.001)$ \\
\hline \multicolumn{5}{|c|}{ Benton Visual Retention Test (VRT) } \\
\hline Match & $-2.8 \pm 2.8$ & $0.0 \pm .94$ & $22 / 21$ & $13.33(.001)$ \\
\hline Delay & $-3.8 \pm 2.8$ & $0.0 \pm .95$ & $21 / 21$ & $39.34(<.001)$ \\
\hline Rosen Drawing Task & $-4.4 \pm 2.5$ & $0.0 \pm .95$ & $26 / 20$ & $46.35(<.001)$ \\
\hline
\end{tabular}

${ }^{a}$ Scores expressed as the difference between normative expectations and current scores, in test score units (e.g., MMS points, IQ points, or IQ subtest scaled score points) for ease of interpretation. Scores for all other tests are expressed as $Z$ scores.

and Category Fluency $(F[1,39]=5.39, p=.026)$, as well as the Rosen Drawing Task $(F[1,39]=7.32, p=.010)$. Occupation effects were found for MMS decline $(F[1,80]=6.36, p=.014)$, the Buschke total recall $(F[1$, $77]=9.42, p=.003), \operatorname{LTR}(F[1,77]=10.49, p=.002), \operatorname{LTR}-\mathrm{B}(F[1$, $77]=11.50, p=.001)$, and Delayed recall $(F[1,75]=4.60, p=.035)$ scores, as well as Cancellation $\operatorname{Six}(F[1,77]=4.56, p=.038)$ and Diamond 
$(F[1,77]=4.56, p=.038)$. Residual variation due to demographic factors could still be extracted, then, despite normative corrections. This may be due to weaknesses in norms themselves (e.g., age correction for the Buschke), absence of norms (e.g., correction for occupational status effects on the MMS), or complex interactions between variables such as age and disease. The existence of these covariance effects provides evidence of the strength of these demographic influences, despite attempts to remove them, that support the rationale for making these corrections.

$r C B F$ indices in AD patients and controls. Both the bilateral PI and the adjusted mean flow differed significantly between $\mathrm{AD}$ patients and controls. Mean PI in AD patients is .92 \pm .058 ; for controls it is $1.01 \pm .036(F[1$, $79]=73.38, p<.001)$. AD patients mean flow is $42.50 \pm 7.94$, while mean flow in controls is $48.87 \pm 7.069 F[1,77]=18.19, p<.001)$. Age, in addition, was found to covary significantly with mean flow $(F[1,77]=5.00$, $p=.028)$. There was moderate overlap between the groups on each measure (36.5\% of AD patients within range of normals for PI; $58.5 \%$ for mean flow), but the central portions of the distributions of each were largely distinct.

Overall regional cerebral perfusion patterns in the patient and control groups are illustrated in Fig. 1. Note that AD patients appear to have deficits in superior as well as inferior parietal cortex (P2 detector sites bilaterally); regions which are not included in the computation of the PI. However, the addition of information from this detector site does not contribute to the discriminability of the PI, nor does it add to its association to neuropsychological measures.

With respect to the laterality of the inferior parietal deficits, the PI appeared worse on the left side. A repeated-measures ANOVA (right- and leftsided PI by diagnosis) yielded a significant group effect, as expected, but also a significant side-by-diagnosis interaction $(F[1,85]=4.01, p<.05)$. The mean difference between the groups (controls minus patients) was .11 on the left side and .07 on the right side. The correlation between right- and left-sided PI was unimpressive. Across all subjects, it was $.50(n=87, p$ $<.0001)$. Within the AD patients it was $.31(n=52, p<.05)$, and within the controls only $.20(n=35, \mathrm{NS})$.

Association of neuropsychological performance to bilateral $r C B F$ indices. Correlations between normatively adjusted neuropsychological measures and both bilateral PI and mean flow are presented in Table 3. These correlations indicate that the bilateral PI predicts a significant amount of the variance in measures of mental status, memory, attention, global intellectual deterioration, and visuoconstructive ability. Associations with intellectual deterioration, as indexed by overall IQ decline and the decline in virtually all IQ subtests, are clearly strongest. Mean flow, however, accounts for a significant degree of variance in IQ decline as well and in one aspect of language skill, Category Fluency.

Measures of virtually all functions that are disturbed in $\mathrm{AD}$, then, are re- 
Alzheimer's Disease Patients $(n=53$, mean flow $=42.5)$
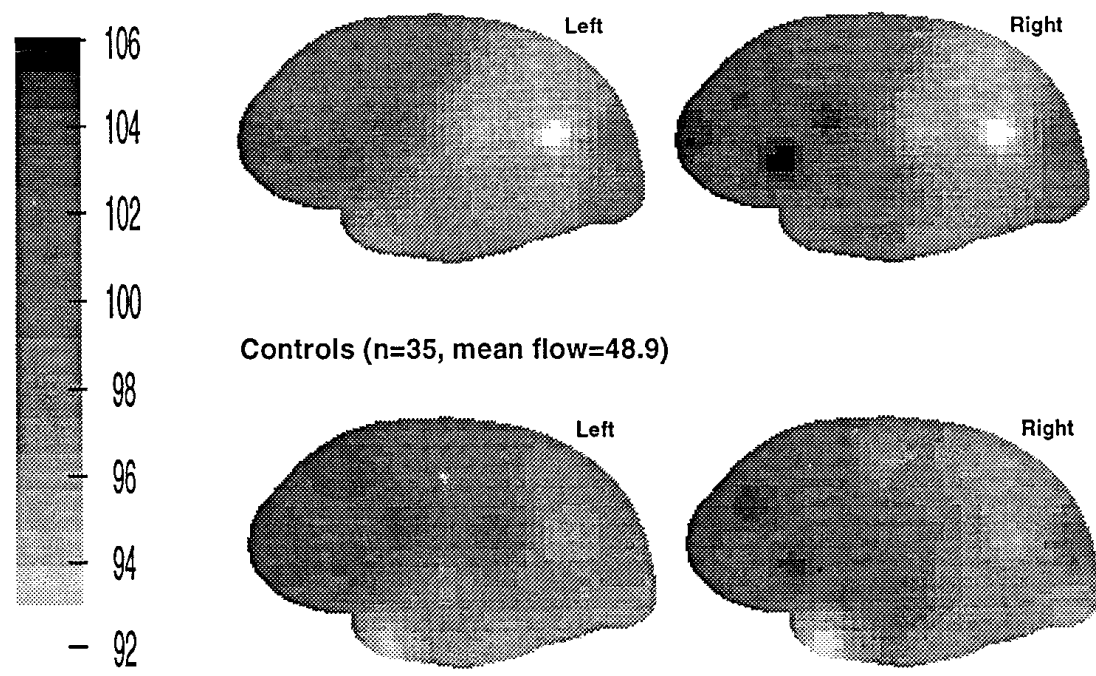

Controls $(n=35$, mean flow $=48.9)$
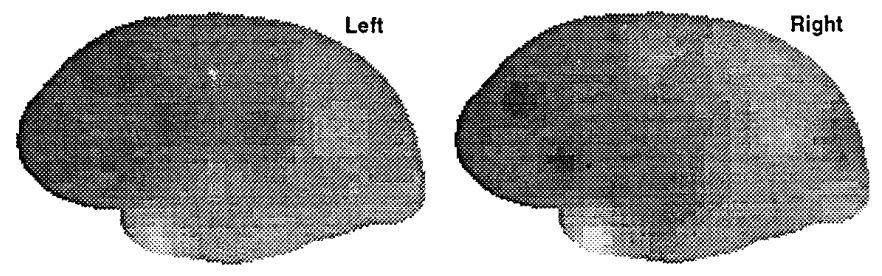

M2 ISI [\%] (relative to total mean)

FIG. 1. Averaged, relative cerebral perfusion patterns (M2 ISI) in AD patient and control subjects. Patient subjects in this sample exhibit the bilateral deficit in parietal perfusion that is characteristic of the disease.

lated to these $\mathrm{rCBF}$ indices. Language measures generally show nonsignificant associations to these $\mathrm{rCBF}$ indices; however, distributional factors may play a role in the weakness of these associations. Because the BDAE total is a composite of subtests scores which are inherently highly negatively skewed (since most normal subjects obtain ceiling scores on most measures), the total score is skewed as well. If the correlation between the PI and BDAE $Z$ score total is recomputed nonparametrically, it becomes highly significant $(r=.62, p<.001$; this is the only association that is changed significantly by using a nonparametric correlation). BDAE subtests for Complex Ideational Material $(r=.57, p<.01)$, Commands $(r=.52, p<.01)$, Primer Level Dictation $(r=.41, p<.05)$, and Responsive Naming $(r=.38, p<.05)$ are also significant nonparametrically.

Because the PI and mean flow are themselves correlated (correlation between PI and mean flow in patient group, $r=.37, p=.006$; long batteryonly group, $r=.48, p=.007$ ) a set of partial correlations was computed between each $\mathrm{rCBF}$ measure and all neuropsychological test scores, controlling for the effects of the other $\mathrm{rCBF}$ measure. This procedure allowed us 
TABLE 3

Correlations between rCBF Indices and Neuropsychological Measures

\begin{tabular}{|c|c|c|c|}
\hline & Parietal index & Mean flow & $N$ 's \\
\hline Mini-Mental State Decline & $.39 * *$ & .09 & $51 / 52$ \\
\hline \multicolumn{4}{|l|}{ Buschke Selective Reminding Test (SRT) } \\
\hline Total & $.36^{*}$ & .12 & $49 / 50$ \\
\hline LTR & $.34 *$ & .09 & $49 / 50$ \\
\hline LTR-B & $.34 *$ & .06 & $49 / 50$ \\
\hline Intrusions & .04 & -.01 & $49 / 50$ \\
\hline Delay & $.31 *$ & .10 & $47 / 48$ \\
\hline Recognition & .07 & .04 & $47 / 48$ \\
\hline \multicolumn{4}{|l|}{ Cancellation Tasks } \\
\hline Six & -.28 & .09 & $38 / 39$ \\
\hline Diamond & $-.40 *$ & .18 & $37 / 38$ \\
\hline TMX & $-.32 *$ & .16 & $37 / 38$ \\
\hline IQ decline, full scale & $.66 * * *$ & $.68 * * *$ & $27 / 27$ \\
\hline IQ decline, verbal scale & $.59 * * *$ & $.65 * * *$ & $27 / 27$ \\
\hline IQ decline, performance scale & $.71 * * *$ & $.68 * * *$ & $27 / 27$ \\
\hline \multicolumn{4}{|l|}{ IQ subtests (decline) } \\
\hline Information & .36 & $.44 *$ & $27 / 27$ \\
\hline Digit Span & $.59 * * *$ & $.56 * *$ & $27 / 27$ \\
\hline Vocabulary & .30 & $.40 *$ & $27 / 27$ \\
\hline Arithmetic & $.59 * * *$ & $.59 * * *$ & $28 / 28$ \\
\hline Comprehension & $.69 * * *$ & $.70 * * *$ & $27 / 27$ \\
\hline Similarities & $.50 * *$ & $.51^{* *}$ & $27 / 27$ \\
\hline Picture Completion & $.58 * *$ & $.63 * * *$ & $27 / 27$ \\
\hline Picture Arrangement & $.55^{* *}$ & $.52 * *$ & $27 / 27$ \\
\hline Block Design & $.67 * * *$ & $.63 * * *$ & $28 / 28$ \\
\hline Object Assembly & $.59 * * *$ & $.57 * * *$ & $28 / 28$ \\
\hline Digit Symbol & $.73 * * *$ & $.55^{* *}$ & $28 / 28$ \\
\hline $\begin{array}{l}\text { Boston Diagnostic Aphasia Exam } \\
\text { (average } Z \text { score/subtest) }\end{array}$ & .38 & .28 & $23 / 23$ \\
\hline Letter fluency (CFL) & .02 & .29 & $25 / 25$ \\
\hline Category fluency (animals) & .35 & $.48^{*}$ & $25 / 25$ \\
\hline \multicolumn{4}{|l|}{ Benton Visual Retention Test (VRT) } \\
\hline Match & .29 & .31 & $22 / 22$ \\
\hline Delay & .21 & .15 & $21 / 21$ \\
\hline Rosen Drawing Task & $.47 *$ & .28 & $26 / 26$ \\
\hline
\end{tabular}

$$
\begin{aligned}
* p & <.05 . \\
* * & <.01 . \\
* * * & <.001 .
\end{aligned}
$$

to assess the independent contribution of each rCBF index to cognitive decline. The results of this analysis are presented in Table 4 and are consistent with the zero-order correlations in Table 3 . The only significant change is that mean flow now accounts for some variance in attention measures, although in the opposite of the expected direction (e.g., higher mean flow, poorer attention performance). In general, however, the core neuropsycho- 
TABLE 4

Partial Correlations between rCBF Indices and Neuropsychological Measures Controlling for Effect of Other rCBF Index

\begin{tabular}{|c|c|c|c|}
\hline & Parietal index & Mean flow & $N$ 's \\
\hline Mini-Mental State Decline & $.38 * *$ & -.06 & $51 / 51$ \\
\hline \multicolumn{4}{|c|}{ Buschke Selective Reminding Test (SRT) } \\
\hline Total & $.34 * *$ & .00 & $49 / 49$ \\
\hline LTR & $.33^{*}$ & -.02 & $49 / 49$ \\
\hline LTR-B & $.34 * *$ & -.06 & $49 / 49$ \\
\hline Intrusions & .05 & -.02 & $49 / 49$ \\
\hline Delay & $.29 *$ & .00 & $49 / 49$ \\
\hline Recognition & .06 & .01 & $49 / 49$ \\
\hline \multicolumn{4}{|l|}{ Cancellation Tasks } \\
\hline Six & $-.32 *$ & .19 & $38 / 38$ \\
\hline Diamond & $-.48 * * *$ & $.34 *$ & $37 / 37$ \\
\hline TMX & $-.39 * *$ & $.28 *$ & $37 / 37$ \\
\hline IQ decline, full scale & $.51^{* *}$ & $.55^{* *}$ & $24 / 24$ \\
\hline IQ decline, verbal scale & $.41 *$ & $.51 * *$ & $24 / 24$ \\
\hline IQ decline, performance scale & $.60 * * *$ & $.54 * *$ & $24 / 24$ \\
\hline \multicolumn{4}{|l|}{ IQ subtests (decline) } \\
\hline Information & .18 & .33 & $24 / 24$ \\
\hline Digit Span & $.43^{*}$ & $.39 *$ & $24 / 24$ \\
\hline Vocabulary & .13 & $.30 *$ & $24 / 24$ \\
\hline Arithmetic & $.43^{*}$ & $.43 *$ & $25 / 25$ \\
\hline Comprehension & $.55^{* *}$ & $.57 * * *$ & $24 / 24$ \\
\hline Similarities & $.33^{*}$ & $.36^{*}$ & $24 / 24$ \\
\hline Picture Completion & $.40^{*}$ & $.48 * *$ & $24 / 24$ \\
\hline Picture Arrangement & $.39 *$ & $.35 *$ & $24 / 24$ \\
\hline Block Design & $.54 * *$ & $.47 * *$ & $25 / 25$ \\
\hline Object Assembly & $.43 *$ & $.41 *$ & $25 / 25$ \\
\hline Digit Symbol & $.64 * * *$ & $.33 *$ & $25 / 25$ \\
\hline $\begin{array}{l}\text { Boston Diagnostic Aphasia Exam } \\
\quad \text { (average } Z \text { score/subtest) }\end{array}$ & .30 & .10 & $23 / 23$ \\
\hline Letter fluency (CFL) & -.12 & .31 & $25 / 25$ \\
\hline Category fluency (animals) & .17 & $.39 *$ & $25 / 25$ \\
\hline \multicolumn{4}{|l|}{ Benton Visual Retention Test (VRT) } \\
\hline Match & .17 & .21 & $22 / 22$ \\
\hline Delay & .16 & .06 & $21 / 21$ \\
\hline Rosen Drawing Task & $.39 *$ & .08 & $26 / 26$ \\
\hline
\end{tabular}

$$
\begin{aligned}
* p & <.05 . \\
* * & <.01 . \\
* * * & <.001 .
\end{aligned}
$$

logical deficits of AD are associated with the PI, while mean flow accounts for declining verbal fluency as well as a substantial degree of variation in global intellectual decline.

Stepwise regression analyses. Stepwise regression was used to find that subset of test scores that could be used to most efficiently predict the bilateral PI. Procedures using all measures from the long battery, however, produced 


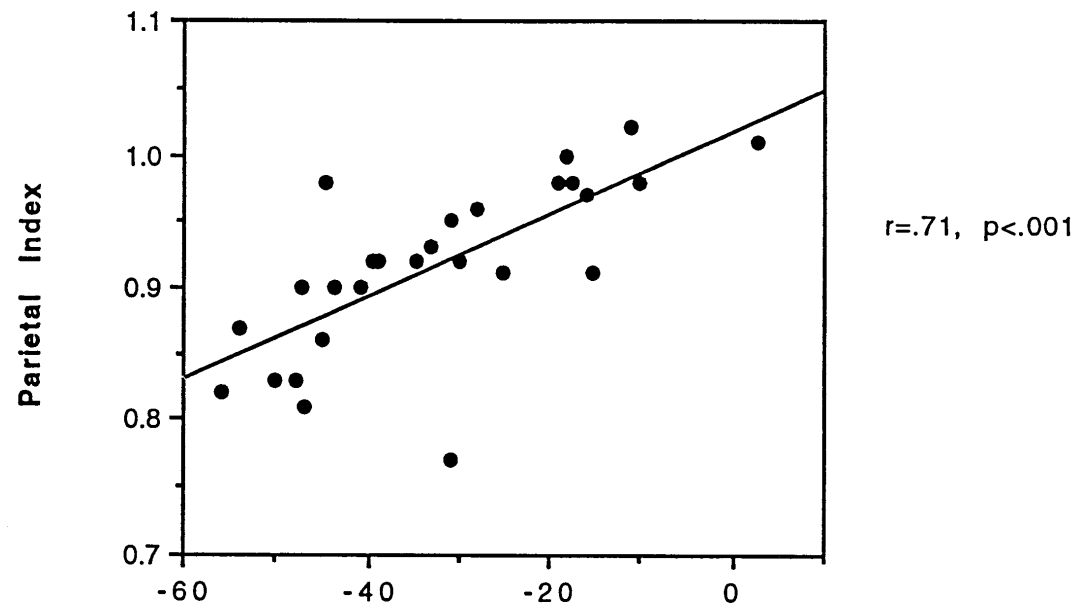

\section{Performance IQ Decline}

FIG. 2. Scatterplot of parietal index vs. the estimated decline in Performance IQ score, for subjects who received the WAIS-R as part of the long neuropsychological battery. The outlying subject exhibits the lowest PI in the sample (as a result of an extremely low value on his right side) but only a moderate Performance IQ decline. With this subject removed $r=.83$ and the regression is highly linear. Note that patient subjects who exhibit less than a 20-point decline in IQ almost all have PI's greater than .95, placing them within the range of normal controls.

an equation with only a single variable, the decline in Performance Scale IQ $\left(R^{2}=.52, F=27.71, p<.0001, n=29\right)$. This equation was obtained even if mean flow was included among the set of predictors. In the analysis of the subset of tests in the short battery, using all subjects, stepwise procedures also produced an equation with only a single predictor, the estimated decline in MMS score $\left(R^{2}=.15, F=8.77, p=.0047, n=53\right)$. This analysis was repeated with only those subjects who received at least one Cancellation Task $(n=38)$ since this task was heavily substituted for in the first short battery run. Results were identical in identifying the MMS as the sole predictor of the PI $\left(R^{2}=.20, F=9.00, p=.0040, n=38\right)$. Note that the MMS is the test from the short battery which is most highly correlated with Performance IQ decline $(r=.59, p=.001)$. Measures of global intellectual decline, then, appear to be the best and most efficient predictors of the PI. A scatterplot of the association between the PI and Performance IQ decline is presented in Fig. 2.

In a supplementary analysis to find those WAIS-R subtests that best predicted the PI, we entered all WAIS-R subtest decline scores into a stepwise regression. Again, however, the final equation included only one predictor variable, the estimated decline in Digit Symbol score $\left(R^{2}=.53, F=31.00\right.$, $p<.0001, n=27$ ). If Digit Symbol is excluded from the set of predictors, 
TABLE 5

Lateralized Parietal Deficits and Neuropsychological Measures

\begin{tabular}{|c|c|c|}
\hline & Left parietal index & Right parietal index \\
\hline Mini-Mental State Decline & .22 & $.26^{*}$ \\
\hline \multicolumn{3}{|c|}{ Buschke Selective Reminding Test (SRT) } \\
\hline Total & -.02 & $.43 * * *$ \\
\hline LTR & .02 & $.38 * *$ \\
\hline LTR-B & .04 & $.38 * *$ \\
\hline Intrusions & .05 & .02 \\
\hline Delay & .05 & $.30 *$ \\
\hline Recognition & $-.28 *$ & $.34 *$ \\
\hline \multicolumn{3}{|l|}{ Cancellation Tasks } \\
\hline Six & $-.34^{*}$ & -.05 \\
\hline Diamond & $-.39 *$ & -.25 \\
\hline TMX & $-.28^{*}$ & -.22 \\
\hline IQ decline, full scale & $.56^{* *}$ & .06 \\
\hline IQ decline, verbal scale & $.52 * *$ & -.04 \\
\hline IQ decline, performance scale & $.58 * * *$ & .18 \\
\hline \multicolumn{3}{|l|}{ IQ subtests (decline) } \\
\hline Information & .26 & -.04 \\
\hline Digit Span & $.56^{* *}$ & -.06 \\
\hline Vocabulary & .30 & -.13 \\
\hline Arithmetic & $.44 * *$ & .08 \\
\hline Comprehension & $.50 * *$ & .20 \\
\hline Similarities & $.57 * * *$ & -.20 \\
\hline Picture Completion & $.37 *$ & .10 \\
\hline Picture Arrangement & $.51 * *$ & -.05 \\
\hline Block Design & $.53 * *$ & .15 \\
\hline Object Assembly & $.50 * *$ & .02 \\
\hline Digit Symbol & $.58 * * *$ & .26 \\
\hline $\begin{array}{l}\text { Boston Diagnostic Aphasia Exam } \\
\text { (average } Z \text { score/subtest) }\end{array}$ & .26 & .07 \\
\hline Letter fluency (CFL) & $.36^{*}$ & $-.44 *$ \\
\hline Category fluency (animals) & $.35^{*}$ & -.16 \\
\hline \multicolumn{3}{|l|}{ Benton Visual Retention Test (VRT) } \\
\hline Match & .35 & -.13 \\
\hline Delay & .33 & -.13 \\
\hline Rosen Drawing Test & .28 & .19 \\
\hline
\end{tabular}

$* p<.05$.
$* * p<.01$.
$* * * p<.001$.

the decline in Comprehension subtest score is the only predictor that is needed in the stepwise equation $\left(R^{2}=.47, F=24.00, p<.0001, n=27\right)$.

Laterality analyses. Partial correlations among parietal indices, computed separately for the left and right hemispheres, and neuropsychological test scores of AD patients are presented in Table 5. Left- and right-sided PI's are found to be associated to different sets of tests when controlling for the 
effects of the PI on the contralateral side and mean flow. Left-sided deficits predict poor performance on measures of attention, intellectual decline, and language fluency. Right-sided deficits are associated with general decline in mental status as well as, surprisingly, deficits in verbal memory performance. The left PI shows a significant association to the Recognition memory subscore of the Buschke, but in the opposite of expected direction. The right PI similarly is correlated with the Letter Fluency score, but in the opposite of the expected direction.

Many fewer test scores were associated with the right-sided PI; however, there is some indication that associations with the right PI were affected by an influential outlying value. This outlying value (very low bilateral PI, lowest right PI, with largest degree of hemispheric asymmetry in sample) significantly attentuated the association to mental status score decline $(r=.37$, $p=.005$ with outlier removed), and suppressed a variety of associations to IQ scores. With the outlier removed, the right PI was significantly correlated with the decline in Full Scale IQ $(r=.42, p=.021)$ and the decline in Performance Scale IQ $(r=.48, p=.009)$. It was also associated to the decline in subtest scores for Arithmetic $(r=.45, p=.015)$, Picture Completion $(r=.53, p=.004)$, Object Assembly $(r=.36, p=.042)$, and Digit Symbol ( $r=.43, r=.017)$. A marginal association was observed for Comprehension $(r=.34, .051)$. No other notable outlier effects were observed.

Overall, these laterality analyses suggest that left-sided inferior parietal deficits carry the greatest significance for deficits in attention, general intellectual performance, and loss of language fluency, while right-sided deficits contribute to general mental status decline, a probable decline in performance-type intellectual skills, and memory dysfunction.

\section{DISCUSSION}

The bilateral PI explained a significant amount of variance in measures of virtually all the neuropsychological functions that are typically disturbed in AD. Thus, this measure has significant value for establishing the degree of overall clinical deterioration that occurs in this disease. Much of the variance in overall neuropsychological impairment, however, could be predicted from the left-sided index of perfusion deficit alone. These associations may reflect the largely "verbal" nature of the neuropsychological tests used here, in the sense that subjects are required to understand instructions and interact with an examiner in order to perform them. These associations may also have been strengthened by the relatively greater severity of left-sided vs. right-sided deficits in this sample. Nonetheless, the greatest degree of variance in neuropsychological data is predicted by left-sided inferior parietal perfusion.

Can we then conclude that left-sided perfusion deficits carry most or all of the significant variance related to intellectual decline in AD? No, because 
right-sided deficits are clearly associated with decrements in general intellectual performance skills, although with aspects of performance that appear to be less verbal in nature. As in one prior study (Robert et al., 1992), the rightsided PI best predicted decline in the Mini-Mental State Exam, a heterogenous measure of memory and praxis, which is sensitive to a variety of impairments in $\mathrm{AD}$ but not very specific. When an outlying value is taken into account, the right-sided PI also predicted deficits in set of IQ subtests involving manipulation of nonverbal materials. In a supplementary analysis not reported above, the right-sided PI best predicted scores on the BDRS ( $r=$ $-.58, p=.009)$ - which measures problems in daily living rather than performance on an interactive test-much better than the left-sided PI ( $r=$ $-.22, p=.206)$ among short duration of illness patients. Thus, the rightsided PI appears to carry significant information about declines in general functional capability, particularly as it is expressed in nonverbal activities. This is consistent with findings from patients with unilateral right-sided disease, who most often present with nonverbal apraxias (Postiglione, Lassen, \& Holman, 1993).

Right-sided deficits also predicted poor verbal memory performance in this sample, which is a core feature of AD but most often found to be associated with left-sided functional deficits. Most likely this is a statistical artifact associated with our subject selection criteria: subjects were diagnosed as probable AD largely as a result of their verbal memory deficits, attenuating the range of verbal memory test scores in the patient group. While a PI at the lowest end of the normal control range (100\% specific) could be used to identify AD patients with a sensitivity of $65 \%$, a Buschke score at the lowest end of the control range (100\% specific) has a sensitivity of $98 \%$. On the other hand, the association between Buschke scores, as they are, and right-sided PI may reflect a deterioration of practical performance skill that is not completely synonymous with verbal memory capacity. Though Buschke SRT scores in the patient sample already reflect a universal impairment of verbal memory, some variation remains, and this "residual" variation may be related to a variety of aspects of brain functioning that reflect the individual's capacity to compensate for their deficits. Data from electrophysiological studies of performance on lateralized cognitive tasks, for example, suggest that performance skill over and above basic competency can often be attributed to contralateral activation, which indicates the degree to which subjects are recruiting additional processing resources to perform a complex task (Moeller, Luber, \& Sackeim, 1993). In this study, the contribution of variability in right-sided inferior parietal perfusion to performance on the Buschke may similarly reflect the degree to which subjects are able to use compensatory strategies, and the degree to which these strategies fail as perfusion to this region declines. Such an interpretation is consistent with studies suggesting that symptomology will be least equivocal when deficits are bilateral (Holman et al., 1992). It is limited, though, by the inherent prob- 
lems of using resting measures of perfusion deficit to characterize dynamic aspects of performance.

Other factors may have contributed to the particular pattern of results observed here. For example, the strength of the association between IQ decline and perfusion deficits, vis-a-vis its association to other neuropsychological measures, may be a function of the superior psychometric qualities of the WAIS-R. Because most neuropsychological instruments other than the WAIS-R have been designed primarily to distinguish dichotomous groups of impaired and unimpaired patients on a particular cognitive function, less attention is often paid to characterizing the full range of ability levels from mildly impaired to superior performance. When compared with the WAIS-R, with its huge normative population, careful scaling across a wide range of ability levels, and normally distributed scores, single function neuropsychological tests are likely to produce less consistent data in analyses based on parametric correlations. This is particularly the case for nonverbal and visual-spatial measures. Some attempt was made to compensate for these limitations here by normatively adjusting scores, but even these procedures cannot completely compensate for all deficiencies of scaling.

The lack of full-battery data for all of our subjects also limited the power of our regression analyses to detect those test scores that predicted some portion of the variance in $\mathrm{rCBF}$ measures, but less than the WAIS-R. This may create the false impression that localized perfusion deficits predict nonspecifically to intellectual decline. Tests like the Performance IQ or MiniMental State clearly are most sensitive to perfusion deficits, but, based on data from this study, it would be premature to conclude that the decline in other functions is simply a consequence of a general loss of intellectual capability.

Our selection of a specific $\mathrm{rCBF}$ index of AD-related pathology, though robust and well-validated, may have also distorted the relationships observed with test data, particularly since it is based on a two-dimensional imaging modality. Alexander, Moeller, Grady, Pietrini, Mentis, and Schapiro (1994b), for example, found that covariance patterns in 18-FDG PET data presumably related to the activity of underlying cortical and cortical/subcortical "networks" could be associated to relatively independent patterns of cognitive loss in $\mathrm{AD}$ subjects. One network involving lowered bilateral parietal metabolism and increased metabolism in the thalamus, lenticular nucleus, anterior cingulate, and orbitofrontal regions was associated with the loss of visuospatial and attentional skills. Another involving reduced inferior parietal metabolism on the left side, along with a variety of other specifically left-sided frontotemporal deficits was associated with loss of verbal memory, language comprehension, and verbal fluency. One difficulty in replicating this finding with planar techniques is the lack of information about cerebral activity below the cortical surface, although similar, multivariate analytic strategies have been used successfully in planar rCBF studies to distinguish 
AD patients from other diagnostic groups (Sackeim et al., 1993). The purpose of this study was to account for that variance in neuropsychological performance related to the most obvious source of differences between AD patients and controls, but other brain regions that are affected by the disease are likely to influence the pattern and course of neuropsychological impairment. In part, this study demonstrates some of the limitations inherent in trying to characterize specific behavioral deficits in terms of simple measures of resting cortical pathology.

Despite these limitations, the simple index of inferior parietal perfusion deficits used in this study predicted general intellectual deterioration very well, raising important questions about both the nature and the timing of these deficits. With respect to their nature, results here suggest that perfusion deficits are most valuable as pathophysiological indices of deterioration from baseline. Clearly, our strategy of correcting scores for normative expectations had a uniformly positive effect on our ability to detect significant associations with imaging data. Normative adjustments were not always perfect, as covariance analyses comparing patient and control performance on many tests revealed, but invariably improved associations to $\mathrm{rCBF}$ data. The positive effect of these normative corrections provides further support for Katzman's position (Katzman, 1993) that the best behavioral indicators of pathological severity are those that assess individual decline from premorbid baseline functioning. It also suggests that normative corrections should be routine in these types of studies in order to at least partially control for the effects of individual premorbid ability levels on test performance. Given these types of adjustments, close associations between pathophysiological severity and measures of functional decline can be demonstrated. The latter can then be used as valuable epidemiological tools in studies where demographic data and cognitive screening measures are available, but brain imaging is not feasible.

With respect to the timing of the appearance of parietal deficits in $\mathrm{AD}$, data here are cross-sectional, but suggest that they may not be apparent until after cognitive symptoms have progressed significantly. Among those patients in this study who had less than an estimated 20-point decline in IQ, $87.5 \%$ had PI's that fell within the range of normal controls (see Fig. 2). Though Holman et al. (1992) suggest that biparietal deficits can be observed in the earliest stages of $\mathrm{AD}$ and may even be predictive of disease before the onset of symptoms, findings here suggest that these deficits will be accompanied by global intellectual decline, which typically follows the loss of mnestic functions (Grady, Haxby, Horwitz, Sundaram, Berg, Schapiro, Friedland, \& Rapoport, 1988). Some of the apparent discrepancy between these findings may be a function of different methods used to assess rCBF, in that Holman et al. used SPECT, which may be more sensitive to parietal abnormalities than the planar methods used here. Data here, though, suggest that significant intellectual decline will have already taken place by the time 
these abnormalities are evident on the cortical surface. Further research, including longitudinal studies of very early stage patients or at-risk samples, is required to more fully characterize these time-course issues.

To summarize, findings here suggest that the characteristic perfusion deficits of $\mathrm{AD}$ correlate very well with its neuropsychological deficits, and that the pathophysiological severity of AD can be observed directly in declining cognitive performance. Deterioration measures derived from the WAIS-R are clearly the most sensitive correlates of perfusion deficit, although some baseline adjustment is necessary in order to maximize these associations. Left and right-sided deficits predicted different patterns of neuropsychological impairment, with left-sided deficits appearing to be more important for the prediction of general cognitive decline. Those functions related to right-sided deficit, however, may not have been assessed very thoroughly here. Performance Scale IQ measures produced the highest associations to perfusion deficit severity, consistent with studies suggesting that relative deterioration of Performance Scale abilities is a hallmark of the disease (see Alexander et al., 1994a). High correlations between the Performance IQ decline measure and parietal perfusion measures appear to result, though, from its sensitivity to deficits on both sides of the brain.

\section{REFERENCES}

Alexander, G. E., Prohovnik, I., Stern, Y., \& Mayeux, R. 1994a. WAIS-R subtest profile and cortical perfusion in Alzheimer's disease. Brain and Cognition, 24, 24-43.

Alexander, G., Moeller, J. R., Grady, C. L., Pietrini, P., Mentis, M. J., \& Schapiro, M. B. 1994b. Association of cognitive functions with regional networks of brain metabolism in Alzheimer's disease. Neurobiology of Aging, 15(Suppl. 1), S36.

Barona, A., Reynolds, C. R., \& Chastain, R. 1984. A demographically based index of premorbid intelligence for the WAIS-R. Journal of Consulting and Clinical Psychology, 52(5), 885-887.

Berkman, L. F. 1986. The association between educational attainment and mental status examination: Of etiological significance for senile dementias or not? Journal of Chronic Disease, 39, 171-174.

Blessed, G., Tomlinson, B. E., \& Roth, M. 1968. The association between quantitative measures of dementia and senile changes in the cerebral grey matter of elderly subjects. British Journal of Psychiatry, 114, 797-811.

Crum, R. M., Anthony, J. C., Bassett, S. S., \& Folstein, M. F. 1993. Population-based norms for the Mini-Mental State examination by age and education level. Journal of the American Medical Association, 269, 2386-2391.

DeKosky, S. T., Shih, W-J., Schmitt, F. A., Coupal, J., \& Kirkpatrick, C. 1990. Assessing utility of single photon emission computed tomography (SPECT) scan in Alzheimer disease: Correlation with cognitive severity. Alzheimer Disease and Associated Disorders, 4(1), 14-23.

DeLeon, M. J., Ferris, S. H., George, A. E., Christman, D. R., Fowler, J. S., Gentes, C., Reisberg, B., Gee, B., Emmerich, M., Yonekura, Y., Brodie, J., Kricheff, I. I., \& Wolf, A. P. 1983. Positron emission tomographic studies of aging and Alzheimer disease. American Journal of Neurological Research, 4, 568-571.

Folstein, M. F., Folstein, S. E., \& McHugh, P. R. 1981. “Mini-Mental State'’: A practical 
method for grading the cognitive state of patients for the clinician. Journal of Psychiatric Research, 12, 189-198.

Frackowiak, R. S. J., Pozzilli, C., Legg, N. J., DuBoulay, G. H., Marshall, J., Lenzi, G. L., \& Jones, T. 1981. Regional cerebral oxygen supply and utilization in dementia: A clinical and physiological study with oxygen-15 and positron tomography. Brain, 104, 753778 .

Friedland, R. A., Budinger, T. F., Ganz, E., Yano, Y., Mathis, C. A., Koss, B., Ober, B. A., Huesman, R. H., \& DeRenzo, S. E. 1983. Regional cerebral metabolic alterations in dementia of the Alzheimer type: Positron emission tomography with $\left[{ }^{18} \mathrm{~F}\right]$ flourodeoxyglucose. Journal of Computer Assisted Tomography, 7, 590-598.

Fuld, P. A. 1984. Test profile of cholinergic dysfunction and Alzheimer's type dementia. Journal of Clinical Neuropsychology, 6, 380-392.

Grady, C. L., Haxby, J. V., Horwitz, B., Sundaram, M., Berg, G., Schapiro, M., Friedland, R. P., \& Rapoport, S. I. 1988. Longitudinal study of the early neuropsychological and cerebral metabolic changes in dementia of the Alzheimer type. Journal of Clinical and Experimental Neuropsychology, 10(5), 576-596.

Holman, B. L., Johnson, K. A., Gerada, B., Carvalho, P. A., \& Satlin, A. 1992. The scintigraphic appearance of Alzheimer's disease. Journal of Nuclear Medicine, 33, 181-185.

Jagust, W. J., Budinger, T. F., \& Reed, B. R. 1987. The diagnosis of dementia with single photon emission computed tomography. Archives of Neurology, 44, 258-262.

Johnson, K. A., Holman, B. L., Mueller, S. P., Rosen, J., English, R., Nagel, J. S., \& Growdon, J. H. 1988. Single photon emission computed tomography in Alzheimer disease: Abnormal iofetamine I 123 reflects dementia severity. Archives of Neurology, 45, 392396.

Karbe, H., Kertesz, A., Davis, J., Kemp, B. J., Prato, F. S., \& Nicholson, R. L. 1994. Quantification of functional deficit in Alzheimer's disease using a computer-assisted mapping program for ${ }^{99 m}$ Tc-HMPAO SPECT. Diagnostic Neuroradiology, 36, 1-6.

Katzman, R. 1993. Education and the prevalence of dementia and Alzheimer's disease. Neurology, 43, 13-20.

Keilp, J. G., \& Prohovnik, I. 1995. Intellectual decline predicts the parietal perfusion deficit in Alzheimer's disease. Journal of Nuclear Medicine, 36(8), 1347-1354.

Kittner, S. J., White, L. R., Farmer, M. E., Wolz, M., Kaplan, E., Moes, E., Brody, J. A., \& Feinleib, M. 1986. Methodological issues in screening for dementia: The problem of education adjustment. Journal of Chronic Disease, 39, 163-170.

Maximilian, V. A., Prohovnik, I., \& Risberg, J. 1980. Cerebral hemodynamic response to mental activation in normo- and hypercapnia. Stroke, 11, 342-347.

Mayeux, R., Stern, Y., Rosen, J., and Leventhal, J. 1981. Depression, intellectual impairment, and Parkinson's Disease. Neurology, 31, 645-650.

McKhann, G., Drachman, D., Folstein, M., Katzman, R., Price, D., and Stadlan, E. M. 1984. Clinical diagnosis of Alzheimer's Disease: Report of the NINCDS-ADRDA work group under the auspices of the Department of Health and Human Services Task Force on Alzheimer's Disease. Neurology, 34, 939-944.

Moeller, J. R., Luber, B., \& Sackeim, H. A. 1993. With network modelling, topographic EEG predicts memory capacity and RT in continuous performance tasks. Society for Neuroscience Abstracts, 19(Part 2, \#659.7), 1606.

Montaldi, D., Brooks, D. N., McColl, J. H., Wyper, D., Patterson, J., Barron, E., \& McCulloch, J. 1990. Measurements of regional cerebral blood flow and cognitive performance in Alzheimer's disease. Journal of Neurology, Neurosurgery, and Psychiatry, 53, 3338.

Postiglione, A., Lassen, N. A., \& Holman, B. L. 1993. Cerebral blood flow in patients with dementia of the Alzheimer's type. Aging, 5(1), 19-26.

Prohovnik, I., Alexander, G., \& Tatemichi, T. K. 1991. Cortical perfusion in vascular and Alzheimer's dementia. Neurology, 41(Suppl. 1), 358.

Prohovnik, I., Knudsen, E., \& Risberg, J. 1983. Accuracy of models and algorithms for deter- 
mination of fast compartment flow by noninvasive ${ }^{133}$ Xe clearance. In P. Magistretti (Ed.), Functional radionuclide imaging of the brain. New York: Raven Press.

Prohovnik, I., Knudsen, E., and Risberg, J. 1985. Theoretical evaluation and simulation test of the initial slope index for noninvasive rCBF. In H. Hartmann \& S. Hoyer (Eds.), Cerebral blood flow and metabolism measurement. Berlin: Springer-Verlag. Pp. 56-60.

Prohovnik, I., Mayeux, R., Sackheim, H. A., Smith, G., Stern, Y., \& Alderson, P. O. 1988. Cerebral perfusion as a diagnostic marker of early Alzheimer's disease. Neurology, 38, 931-937.

Prohovnik, I. 1988. Data quality, integrity, and interpretation. In S. Knezevic, V. A. Maximillian, Z. Mubrin, I. Prohovnik, \& J. Wade (Eds.), Handbook of regional cerebral blood flow. Hillsdale, NJ: Erlbaum. Pp. 51-78.

Robert, Ph., Migneco, O., Darcourt, J., Ricq, O., Aubin, V., Bonhomme, P., Pringuey, D., Lapalus, F., \& Darcourt, G. 1992. Correlation between ${ }^{99 \mathrm{~m}}$ Tc-HMPAO brain uptake and severity of dementia in Alzheimer's disease: Assessment using an automated technique. Dementia, 3, 15-20.

Rosen, W. 1981. The Rosen Drawing Test. Bronx, NY: Veteran's Administration Medical Center.

Sackeim, H. A., Prohovnik, I., Moeller, J. R., Brown, R. P., Apter, S., Prudic, J., Devanand, D. P., \& Mukherjee, S. 1990. Regional cerebral blood flow in mood disorders. Archives of General Psychiatry, 47, 60-70.

Sackeim, H. A., Prohovnik, I., Moeller, J. R., Mayeux, R., Stern, Y., \& Devanand, D. P. 1993. Regional cerebral blood flow in mood disorders. II. Comparison of major depression and Alzheimer's disease. Journal of Nuclear Medicine, 34, 1090-1101.

Sano, M., Rosen, W., \& Mayeux, R. 1984. Attention deficits in Alzheimer's disease. Presented at the 92nd annual meeting of the American Psychological Association, Toronto, Ontario.

Stern, Y., Alexander, G. E., Prohovnik, I., \& Mayeux, R. 1992. Inverse relationship between education and parietotemporal perfusion deficit in Alzheimer's Disease. Annals of Neurology, 32, 371-375.

Wechsler, D. 1981. Wechsler Adult Intelligence Scale-Revised Manual. Cleveland, OH: Psychological Corporation, Harcourt Brace Jovanovich. 